\title{
Using different surgical techniques and ideas to reduce post-operative adhesion formation: a systematic review and meta-analysis
}

\author{
Rekha Rani*, Shikha Singh, Urvashi, Ruchika Garg
}

Department of Obstetrics and Gynaecology, Sarojini Naidu Medical College, Agra, Uttar Pradesh, India

Received: 15 July 2021

Revised: 08 August 2021

Accepted: 09 August 2021

\section{*Correspondence:}

Dr. Rekha Rani,

E-mail: drrekha.gynae@gmail.com

Copyright: () the author(s), publisher and licensee Medip Academy. This is an open-access article distributed under the terms of the Creative Commons Attribution Non-Commercial License, which permits unrestricted non-commercial use, distribution, and reproduction in any medium, provided the original work is properly cited.

\begin{abstract}
Adhesion development is the most common sequelae of intra-abdominal and pelvic surgery. Using a good surgical technique is advocated as a first step in preventing adhesions. However, the evidence for different surgical techniques to reduce adhesion formation needs confirmation. This review contributed to the growing knowledge pool by elucidating factors that potentially predispose to the development of adhesions. A literature search was performed using the PubMed database for all relevant English language articles and were reviewed with particular attention to predisposing factors to post-operative adhesion development. In addition, the reference lists of each article were reviewed to identify additional relevant articles. Various factors have been shown to directly increase the risk of postoperative adhesion development; namely, certain genetic polymorphisms in the interleukin-1 receptor antagonist, increased estrogen exposure, and endometriosis. There were 28 papers with 27 studies included for a systematic review. Of these, 17 studies were eligible for meta-analysis and 11 for qualitative assessment only. None of the techniques that were compared significantly reduced the incidence of adhesive small bowel obstruction. In a small low-quality trial, the pregnancy rate increased after subserous fixation of suture knots. However, the incidence of adhesions was lower after laparoscopic compared with open surgery (relative risk (RR): 0.14 ; 95\% confidence interval (CI): $0.03-0.61)$ and when the peritoneum was not closed (RR: 0.36 ; $95 \%$ CI: $0.21-0.63$ ). None of the specific techniques that were compared reduced the two main adhesion-related clinical outcomes, small bowel obstruction and infertility.
\end{abstract}

Keywords: Tissue adhesions, Laparoscopy, Peritoneal closure, Infertility, Small bowel obstruction

\section{INTRODUCTION}

Adhesion development is the most common sequelae of intra-abdominal and pelvic surgery and represents a significant cause of morbidity among post-operative patients. The incidence of adhesive small bowel obstruction (SBO) was $2 \%$. Among patients with a known cause of SBO, adhesions were the single most common cause. Using a good surgical technique is advocated as a first step in preventing adhesions. However, the evidence for different surgical techniques to reduce adhesion formation needs confirmation.
Adhesion formation following pelvic surgery is also common and is a major cause of infertility in women. The mechanism by which adhesions cause infertility includes distortion of the normal ability of the fallopian tube to achieve ovum pickup following ovulation, which can be due to ovarian encapsulation by adhesions or limitations in tubal/fimbral potential for movement.

It has been estimated that $22 \%$ of all infertility cases are attributable to adhesions. In one study, adhesions were found in $37 \%$ of infertile patients. In $15 \%$ of these cases, adhesions were the only factor identified as the cause of 
infertility. ${ }^{1}$ Interestingly, pregnancy rates have been shown to increase by 38 to $52 \%$ among previously infertile patients following laparotomy with adhesiolysis, demonstrating the potential value of adhesiolysis.

Another major consequence of adhesion development is chronic pelvic or abdominal pain, likely the result of increased tension on internal organs at sites stretched as a consequence of anomalous attachments. Another major consequence of adhesion development is chronic pelvic or abdominal pain, likely the result of increased tension on internal organs at sites stretched as a consequence of anomalous attachments . Upon laparoscopic adhesiolysis, $74 \%$ of these patients had either a reduction or a complete resolution of their pain, indicating that adhesions were the sole contributor to their pain. ${ }^{2}$ Meta-analysis show an increase in operative time by $15 \mathrm{~min}$ in patients with previous surgery, as well as a reduction in operative time with the placement of an anti-adhesion barrier. ${ }^{3}$

\section{Sequelae of postoperative adhesions}

\begin{tabular}{|c|c|c|c|}
\hline $\begin{array}{c}\text { Adhesive bands } \\
\text { distort bowel loops }\end{array}$ & $\begin{array}{c}\text { Distorted ovum } \\
\text { pickup }\end{array}$ & $\begin{array}{c}\text { Increased Tension } \\
\text { Stretching Serosal } \\
\text { Surfaces }\end{array}$ & $\begin{array}{c}\text { Longer reoperative } \\
\text { time:surgical } \\
\text { complication }\end{array}$ \\
$\begin{array}{c}\text { Small bowel } \\
\text { obstruction }\end{array}$ & Infertility & Chronic Pelvic Pain & $\begin{array}{c}\text { Bowerence to } \\
\text { anterior abdominal } \\
\text { wall }\end{array}$ \\
& & $\begin{array}{c}\text { Increased Healthcare } \\
\text { Expenditure }\end{array}$ & $\begin{array}{c}\text { Bowel injury during } \\
\text { abdominal entry }\end{array}$ \\
\hline
\end{tabular}

Figure 1: Common clinical sequelae of post-operative adhesion.

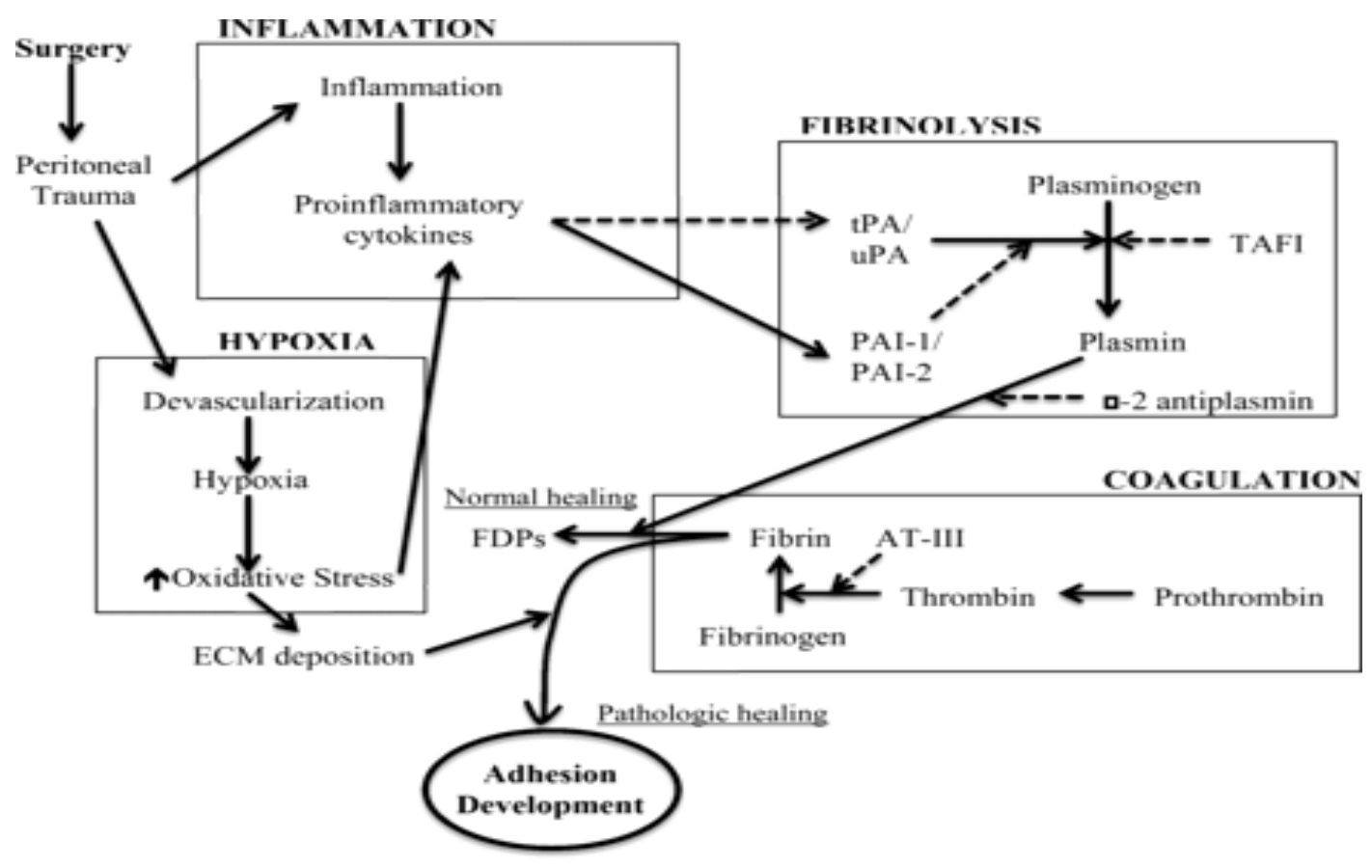

Figure 2: Interconnected processes.

\section{Pathophysiology}

The peritoneum is an extensive layer of mesothelial cells that functions to protect the abdominal organs and reduce friction between their viscera. ${ }^{4}$ The peritoneum is exquisitely delicate and highly susceptible to trauma due to the loose interconnection between mesothelial cells. Damage to the peritoneum can be secondary to inflammatory or surgical causes. Inflammatory damage occurs as a result of intra-abdominal inflammatory 
processes, including pelvic inflammatory disease, possibly past use of an intrauterine contraceptive device. There are a handful of factors that can damage the peritoneum perioperatively. These include trauma, ischemia, infection, exposure to intestinal contents and foreign bodies (e.g. talcum and powders from gloves, fibers from disposable paper items and lint from abdominal packs). Postsurgical adhesions are the most common subtype of adhesions and will be the focus of this review.

Hypoxia and the subsequent oxidative stress is believed to play a significant role in the pathogenesis of post-operative adhesions. In support of this concept, studies suggested that acute oxidative stress in the peritoneum subsequently induces mesothelial cell loss or dysfunction, peritoneal fibrosis and intra-abdominal adhesion formation. During the first $5 \mathrm{~min}$ of ischemia, there is already a significant production of free radicals, either through an increase in reactive oxygen species (ROS) formation or by decreasing ROS scavengers. ${ }^{5,6}$ The enhanced production of ROS is associated with phenotypic changes such as enhanced expression of cytokines, growth factors and extracellular matrix as well as genotypic changes such as alterations in DNA sequence of NAPH oxidase.

Once the peritoneum is damaged, the coagulation cascade is set in motion. The coagulation cascade involves the conversion of a series of inactive proenzymes to active enzymes, ultimately resulting in the formation of a clot. Intrinsic and extrinsic pathways lead to the activation of factor $X$, which then triggers the conversion of prothrombin (factor II) to thrombin (factor IIa). Thrombin serves as the final enzyme of this cascade and converts fibrinogen into fibrin monomers. These fibrin monomers then polymerize to form an insoluble fibrin clot.

This cascade is a normal hemostatic response to tissue injury and is targeted at repair of the damage. However, if two damaged peritoneal surfaces come in contact with each other, the healing process can in essence result in fusion to form a connection, an adhesion.

Research indicated that the coagulation cascade was altered in response to tissue hypoxia. Studies have demonstrated a marked reduction of the tPA/PAI-1 (tissue plasminogen activator/plasminogen activator inhibitor-I) mRNA expression ratio as well as decreased tPA activity in response to tissue hypoxia. Thus the likelihood that fibrinous collections at surgical sites would undergo fibrinolysis was markedly reduced. Subsequent fibroblast migration into the fibrinous mass and deposition of extracellular matrix resulted in adhesion development. Figure 2 depicts the interconnected processes of inflammation, hypoxia, coagulation, and fibrinolysis and their role in the development of post-operative adhesion formation.

Inflammation, hypoxia, coagulation and fibrinolysis and their role in the development of post-operative adhesion formation. Dashed lines represent inhibition. AT-III, antithrombin III; ECM, extracellular matrix; FDPs, fibrin degradation products; PAI, plasminogen activator inhibitor; TAFI, thrombin-activatable fibrinolysis inhibitor; tPA, tissue plasminogen activator; uPA, urokinase-type plasminogen activator.

\section{METHODS}

\section{Search}

A comprehensive literature search was carried out in Pubmed, Embase and Central. A list of predefined search terms was combined with the Cochrane highly sensitive strategy for Pubmed (Table 1). Similar keywords were used for searching Embase and Central. The Embase search was combined with the sensitivity maximizing search strategy described by Wong et al in 2006. No language or date restrictions were applied. The latest search was carried out on 1 October 2011. A manual search of the bibliographies of relevant papers was carried out to identify additional studies for possible inclusion.

Various factors have been shown to directly increase the risk of post-operative adhesion formation. A summary of the factors associated with increased or decreased risk of adhesion development as well as with alterations in fibrosis, is depicted in Table 1. These factors have been grouped into five major categories: surgical and medical history (type of surgery, medications, diabetes mellitus, cancer, endometriosis); reproductive milieu (hormones, menstrual cycle, pregnancy); specific demographics (gender, age, genetics); lifestyle and nutritional factors (obesity, exercise, diet, alcohol, smoking) and psychological well-being (stress, mood). Figure 3 depicts the impact that each of these categories has on the coagulation and fibrinolytic systems with its consequential propensity for adhesion development, or for remesotheliazation and healing without adhesion development, respectively.

\section{Factors associated with increased/decreased risk of fibrosis as well as post-operative adhesion formation}

\section{Hypothesized to increase risk of adhesions}

Medical/surgical history of adhesiolysis, endometriosis, estrogen intake were hypothesized to increase risk of adhesions.

\section{Hypothesized to increase risk of fibrosis}

Anti-Parkinsonian drugs, diabetes mellitus, obesity, cancer, alcohol consumption, smoking were hypothesized to increase tisk of fibrosis.

\section{Unclear or conflicting finding}

Lower GI surgery, menstrual cycle, gender, age were the unclear or conflicting findings. 
Hypothesized to decrease risk of fibrosis

Statin, low-saturated-fat diet, coffee, antioxidants, light to moderate consumption were hypothesized to decrease the risk of fibrosis.

\section{Hypothesized to decrease risk of adhesions}

Tamoxifen was hypothesized to decrease the risk of adhesions.

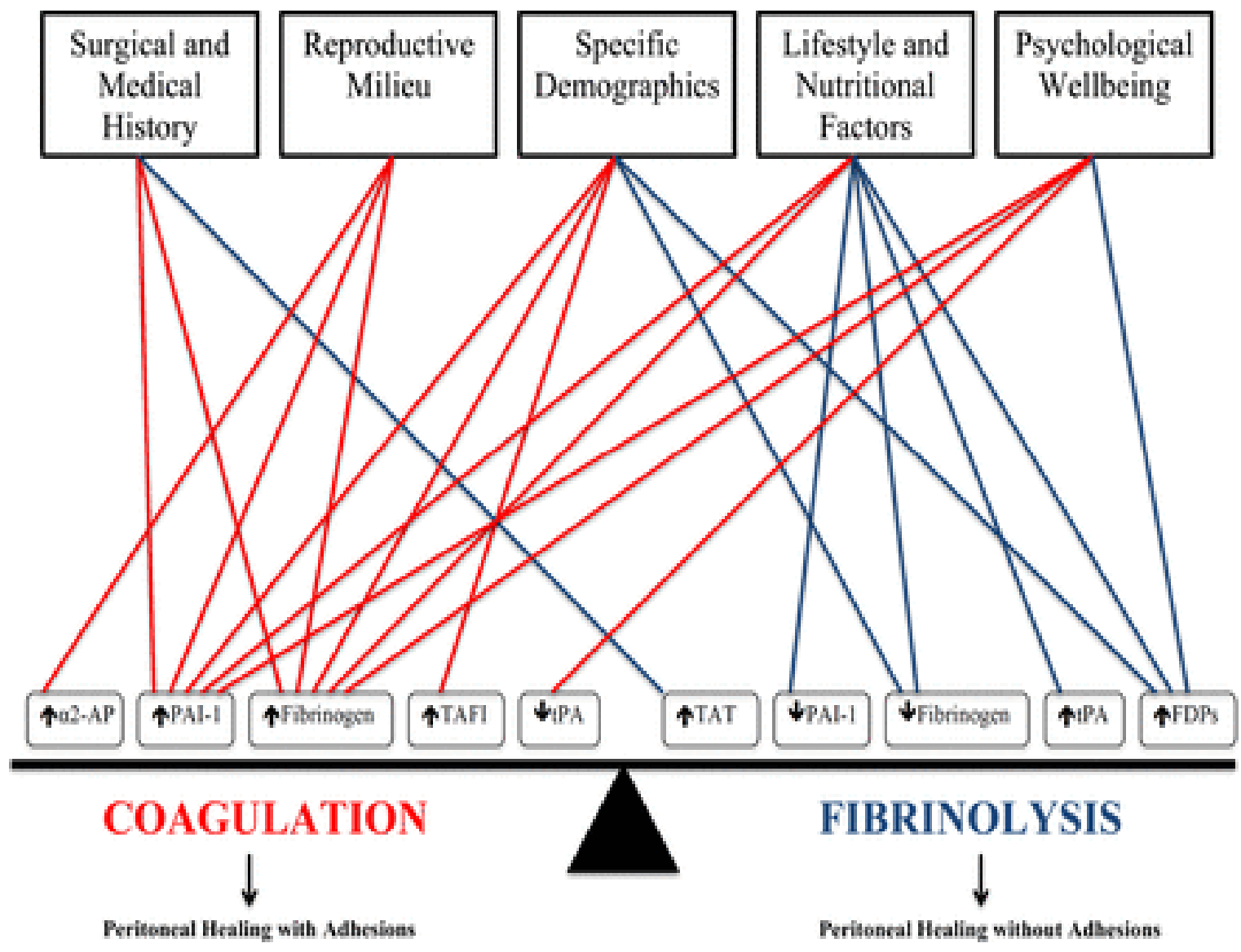

Figure 3: The impact of predisposing factors on key players of the coagulation and fibrinolytic systems; $\alpha 2$-AP, $\alpha 2-$ antiplasmin; TAT, thrombin/antithrombin III complex.

Table 1: Predefined search terms used in Pubmed.

\begin{tabular}{|ll|}
\hline Sr. No. & Predefined search terms (title/abstract) \\
\hline \multicolumn{2}{|c|}{ Patients } \\
\hline 1. & Abdo* \\
\hline 2. & Intraabdominal \\
\hline 3. & Peritoneal \\
\hline 4. & Intraperitoneal \\
\hline 5. & Laparoscop* \\
\hline 6. & Laparotom* \\
\hline 7. & Myomect* \\
\hline 8. & Gyne* \\
\hline 9. & Surgi* \\
\hline 10. & Surge* \\
\hline 11. & Color* \\
\hline 12. & Pelv* \\
\hline 13. & Cesarean section \\
\hline 14. & Caesarean section \\
\hline 15. & Combine 1 or 2 or 3 or 4 or 5 or 6 or 7 or 8 or 9 or 10 or 11 or 12 or 13 or 14 or 15 or 16 \\
\hline & Interventions \\
\hline
\end{tabular}




\begin{tabular}{|ll|}
\hline Sr. No. & Predefined search terms (title/abstract) \\
\hline 16. & Electrocoag* \\
\hline 17. & Electrotherm* \\
\hline 19. & Ultrason* \\
\hline 20. & Harmonic scalpel \\
\hline 21. & Ultracision \\
\hline 22. & Periotneal \\
\hline 23. & Peritoneum \\
\hline 24. & Lavage \\
\hline 25. & Clur* \\
\hline 26. & Powder \\
\hline 27. & Foreign* \\
\hline 28. & Laparoscope \\
\hline 29. & Laparotom* \\
\hline 30. & Hydra \\
\hline 31. & Conditioning \\
\hline 32. & Antibio* \\
\hline 33. & Laser \\
\hline 34. & Combine 18 or 19 or 20 or 21 or 22 or 23 or 24 or 25 or 26 or 27 or 28 or 29 or 30 or 31 or 32 or 33 or 34 or \\
\hline & Control \\
\hline 35. & - Outcome \\
\hline & Adhesi* \\
\hline 36. & Tissue adhesions (mesh terms) \\
\hline 37. & Combine 37 or 38 \\
\hline 37. & Total \\
\hline 38. & Combine 17 and 36 and 39 \\
\hline
\end{tabular}

\section{RESULTS}

\section{Description and quality of included studies}

Searches identified 3912 publications. After removal of duplicates, abstracts and titles of 2854 publications were assessed. There were 59 potentially relevant papers were identified from title and abstract and 31 papers were excluded. One paper was written in Romanian language and full text could not be retrieved. The other 30 papers were excluded from analysis after reading the full text. There were 25 papers excluded because they did not compare different surgical techniques or did not report an adhesion-related outcome. Four papers encompassed studies already included and provided no additional information on outcomes or methodology. Only one paper described a study protocol

Finally, 28 papers describing 27 studies were included (Figure 4). Two papers of Lundorff et al in 1991 and 1992 reported results on different outcomes of the same trial. ${ }^{7}$

All 27 studies were published between 1986 and 2010 and addressed different topics of surgical technique. There were 23 studies performed in patients undergoing gynaecological surgery.

\section{Two techniques of caesarean section}

The study of Nabhan et al on caesarean section was separately analysed because the operative technique between the experimental and control group differed on more aspects than peritoneal closure alone. This study was not suitable for meta-analysis because $79.3 \%$ of patients were lost to follow up by Nabhan et al in 2008 .

In the standard technique control group, caesarean section was performed using the traditional Pfannenstiel-Kerr technique, making a bladder flap and closing the peritoneum. In the modified technique group, the JoelCohen-Stark technique (based on the Misgav Ladach technique) was used, without making a bladder flap and without closing the peritoneum. The incidence of adhesions was significantly lower in the modified technique group ( 11.3 versus $35.5 \%$; $\mathrm{p}=0.003$ ) by Nabhan et al in 2008. Obviously, this reduction cannot solely be attributed to peritoneal non-closure. 


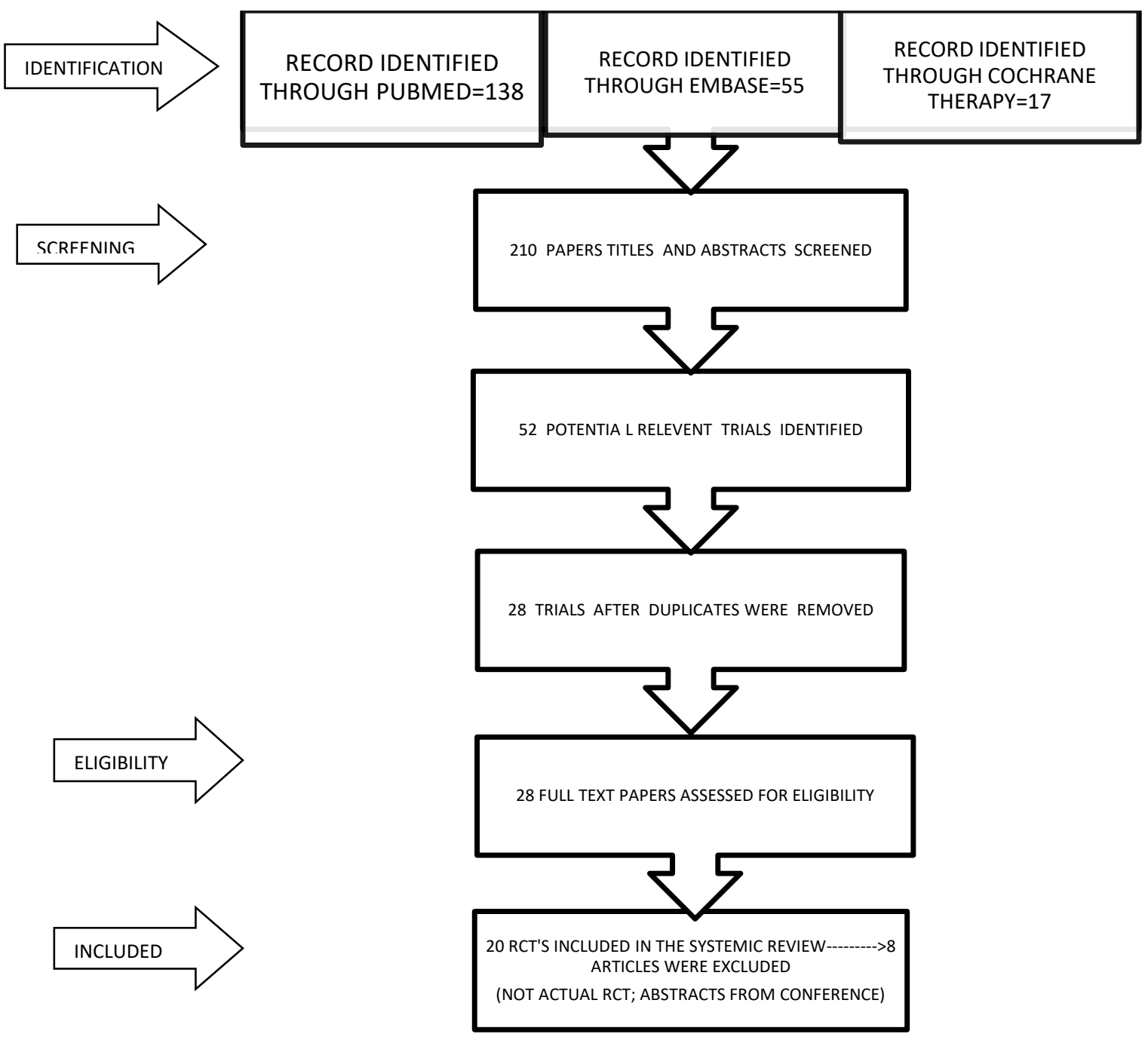

Figure 4: Flow diagram.

Table 2: Risk of bias assessment summary.

\begin{tabular}{|c|c|c|c|c|c|c|c|c|}
\hline References & $\begin{array}{l}\text { Adequ } \\
\text { ate } \\
\text { sequen } \\
\text { ce } \\
\text { genera } \\
\text { tion }\end{array}$ & $\begin{array}{l}\text { Allocatio } \\
\text { n } \\
\text { conceal } \\
\text { ment }\end{array}$ & $\begin{array}{l}\text { Blinding } \\
\text { blinding } \\
\text { (observer) }\end{array}$ & $\begin{array}{l}\text { Adequate } \\
\text { reporting } \\
\text { on loss to } \\
\text { follow up }\end{array}$ & $\begin{array}{l}\text { Power } \\
\text { analysi } \\
\text { s }\end{array}$ & $\begin{array}{l}\text { Free of } \\
\text { other } \\
\text { sources } \\
\text { of bias }\end{array}$ & $\begin{array}{l}\text { Domai } \\
\text { ns with } \\
\text { low } \\
\text { risk of } \\
\text { bias } \\
\text { (n) }\end{array}$ & $\begin{array}{l}\text { Comment-other } \\
\text { source of bias }\end{array}$ \\
\hline $\begin{array}{l}\text { Fujishita et } \\
\text { al (2004) }\end{array}$ & Yes & $\mathrm{Nr}$ & No & No & NR & No & 1 & $\begin{array}{l}\text { Not all randomized } \\
\text { patients had } \\
\text { pregnancy desire; } \\
\text { no fertility analysis } \\
\text { described }\end{array}$ \\
\hline $\begin{array}{l}\text { Franchi et al } \\
\text { (1997) }\end{array}$ & Yes & NR & NR & Yes & Yes & Yes & 4 & \\
\hline $\begin{array}{l}\text { Gurgan et al } \\
\text { (1991) }\end{array}$ & NR & NR & NR & NR & NR & NR & 0 & $\begin{array}{l}\text { Randomization of } \\
\text { patients not } \\
\text { explicitly reported }\end{array}$ \\
\hline $\begin{array}{l}\text { Gurgan et } \\
\text { al (1992) }\end{array}$ & Yes & NR & No & Yes & NR & NR & 2 & $\begin{array}{l}\text { No assessment and } \\
\text { comparison of } \\
\text { adhesion in initial } \\
\text { surgery }\end{array}$ \\
\hline
\end{tabular}

Continued. 


\begin{tabular}{|c|c|c|c|c|c|c|c|c|}
\hline References & $\begin{array}{l}\text { Adequ } \\
\text { ate } \\
\text { sequen } \\
\text { ce } \\
\text { genera } \\
\text { tion } \\
\end{array}$ & $\begin{array}{l}\text { Allocatio } \\
\text { n } \\
\text { conceal } \\
\text { ment }\end{array}$ & $\begin{array}{l}\text { Blinding } \\
\text { blinding } \\
\text { (observer) }\end{array}$ & $\begin{array}{l}\text { Adequate } \\
\text { reporting } \\
\text { on loss to } \\
\text { follow up }\end{array}$ & $\begin{array}{l}\text { Power } \\
\text { analysi } \\
\mathrm{s}\end{array}$ & $\begin{array}{l}\text { Free of } \\
\text { other } \\
\text { sources } \\
\text { of bias }\end{array}$ & $\begin{array}{l}\text { Domai } \\
\text { ns with } \\
\text { low } \\
\text { risk of } \\
\text { bias } \\
\text { (n) }\end{array}$ & $\begin{array}{l}\text { Comment-other } \\
\text { source of bias }\end{array}$ \\
\hline $\begin{array}{l}\text { Kadanali et } \\
\text { al (1996) }\end{array}$ & NR & Yes & Yes & Yes & NR & NR & 3 & $\begin{array}{l}\text { Length till second } \\
\text { look operation not } \\
\text { described }\end{array}$ \\
\hline $\begin{array}{l}\text { Kapustian et } \\
\text { al (2011) }\end{array}$ & Yes & NR & Yes & No & Yes & No & 3 & $\begin{array}{l}\text { Repeat Caesarean } \\
\text { section might } \\
\text { introduce selection } \\
\text { bias }\end{array}$ \\
\hline $\begin{array}{l}\text { Komoto et } \\
\text { al (2006) }\end{array}$ & No & No & No & No & NR & No & 0 & $\begin{array}{l}\text { Repeat Caesarean } \\
\text { section might } \\
\text { introduce selection } \\
\text { bias }\end{array}$ \\
\hline $\begin{array}{l}\text { Lundorff } \\
(\mathbf{1 9 9 1 + 1 9 9 2 )}\end{array}$ & Yes & Yes & No & No & NR & No & 2 & $\begin{array}{l}\text { Second look not } \\
\text { planned for all } \\
\text { randomized } \\
\text { patients; Not all } \\
\text { randomized } \\
\text { patients had } \\
\text { pregnancy desire }\end{array}$ \\
\hline $\begin{array}{l}\text { Malvasi et } \\
\text { al (2009) }\end{array}$ & No & No & No & Yes & NR & NR & 1 & $\begin{array}{l}\text { Repeat Caesarean } \\
\text { section might } \\
\text { introduce selection } \\
\text { bias }\end{array}$ \\
\hline $\begin{array}{l}\text { Mercorio et } \\
\text { al (2008) }\end{array}$ & Yes & Yes & No & Yes & NR & Yes & 4 & \\
\hline $\begin{array}{l}\text { Nabhan et al } \\
(2008)\end{array}$ & Yes & Yes & No & No & Yes & No & 3 & $\begin{array}{l}\text { Repeat Caesarean } \\
\text { section might } \\
\text { introduce selection } \\
\text { bias }\end{array}$ \\
\hline $\begin{array}{l}\text { Ng et al } \\
(2009)^{\mathrm{a}}\end{array}$ & Yes & Yes & No & Yes & Yes & Yes & 5 & \\
\hline $\begin{array}{l}\text { Pellicano et al } \\
(2005)\end{array}$ & No & NR & No & Yes & NR & No & 1 & $\begin{array}{l}\text { No fertility } \\
\text { analysis described }\end{array}$ \\
\hline $\begin{array}{l}\text { Pellicano et al } \\
(\mathbf{2 0 0 8 )}\end{array}$ & Yes & NR & Yes & Yes & NR & No & 3 & $\begin{array}{l}\text { Problems with } \\
\text { statistical analysis }\end{array}$ \\
\hline $\begin{array}{l}\text { Roy et al } \\
(2009)\end{array}$ & NR & NR & NR & Yes & NR & No & 1 & $\begin{array}{l}\text { Second look only } \\
\text { in patients who } \\
\text { failed to conceive, } \\
\text { excluded for } \\
\text { outcome second } \\
\text { look }\end{array}$ \\
\hline $\begin{array}{l}\text { Sharma et } \\
\text { al (2006) }\end{array}$ & Yes & NR & NR & Yes & NR & No & 2 & $\begin{array}{l}\text { Fertility analysis } \\
\text { described is } \\
\text { incomplete; } \\
\text { Pregnancy rate in } \\
\text { abstract does not } \\
\text { correspond to rate } \\
\text { in full text. }\end{array}$ \\
\hline $\begin{array}{l}\text { Stocchi et al } \\
(2008)\end{array}$ & NR & NR & NR & Yes & NR & No & 1 & $\begin{array}{l}\text { No prospective } \\
\text { follow-up; might } \\
\text { have included } \\
\text { some patients } \\
\text { before start of } \\
\text { randomization }\end{array}$ \\
\hline
\end{tabular}




\begin{tabular}{|c|c|c|c|c|c|c|c|c|}
\hline References & $\begin{array}{l}\text { Adequ } \\
\text { ate } \\
\text { sequen } \\
\text { ce } \\
\text { genera } \\
\text { tion }\end{array}$ & $\begin{array}{l}\text { Allocatio } \\
\text { n } \\
\text { conceal } \\
\text { ment }\end{array}$ & $\begin{array}{l}\text { Blinding } \\
\text { blinding } \\
\text { (observer) }\end{array}$ & $\begin{array}{l}\text { Adequate } \\
\text { reporting } \\
\text { on loss to } \\
\text { follow up }\end{array}$ & $\begin{array}{l}\text { Power } \\
\text { analysi } \\
\text { S }\end{array}$ & $\begin{array}{l}\text { Free of } \\
\text { other } \\
\text { sources } \\
\text { of bias }\end{array}$ & $\begin{array}{l}\text { Domai } \\
\text { ns with } \\
\text { low } \\
\text { risk of } \\
\text { bias } \\
\text { (n) }\end{array}$ & $\begin{array}{l}\text { Comment-other } \\
\text { source of bias }\end{array}$ \\
\hline $\begin{array}{l}\text { Takahashi et } \\
\text { al (2007) }\end{array}$ & NR & NR & No & Yes & NR & Yes & 2 & $\begin{array}{l}\text { Randomization of } \\
\text { patients not } \\
\text { explicitly reported }\end{array}$ \\
\hline $\begin{array}{l}\text { Talwar et } \\
\text { al (1997) }\end{array}$ & No & No & No & NR & NR & No & 0 & $\begin{array}{l}\text { Criteria for clinical } \\
\text { diagnosis of ASBO } \\
\text { not given }\end{array}$ \\
\hline $\begin{array}{l}\text { Taskin et } \\
\text { al (1999) }\end{array}$ & Yes & NR & NR & NR & NR & No & 1 & $\begin{array}{l}\text { Inconsistencies in } \\
\text { reported outcomes }\end{array}$ \\
\hline $\begin{array}{l}\text { Alborzi et al } \\
(2003)\end{array}$ & Yes & NR & No & No & NR & No & 1 & $\begin{array}{l}\text { Adhesion score } \\
\text { used is highly } \\
\text { subjective }\end{array}$ \\
\hline $\begin{array}{l}\text { Eshuis et al } \\
(2010)^{a}\end{array}$ & Yes & Yes & No & No & Yes & Yes & 4 & \\
\hline $\begin{array}{l}\text { Weerawetwat } \\
\text { et al }(2004)\end{array}$ & NR & NR & Yes & No & NR & No & 1 & $\begin{array}{l}\text { Repeat Caesarean } \\
\text { section might } \\
\text { introduce selection } \\
\text { bias }\end{array}$ \\
\hline $\begin{array}{l}\text { Taylor et } \\
\text { al (2010) }\end{array}$ & Yes & Yes & NR & No & Yes & No & 3 & $\begin{array}{l}\text { No prospective } \\
\text { follow-up }\end{array}$ \\
\hline $\begin{array}{l}\text { Zareian et al } \\
(2006)\end{array}$ & Yes & Yes & NR & No & NR & No & 2 & $\begin{array}{l}\text { Repeat Caesarean } \\
\text { section might } \\
\text { introduce selection } \\
\text { bias }\end{array}$ \\
\hline
\end{tabular}

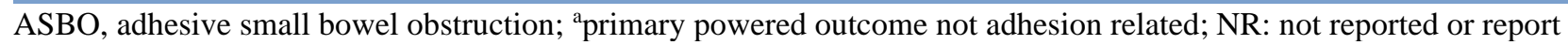
insufficient for judgement.

Table 3: Characteristics of trials included in meta-analysis.

\begin{tabular}{|c|c|c|c|c|c|c|c|}
\hline Study & Period & Patients & Interventions & $\mathbf{N}$ & Outcomes & $\begin{array}{l}\text { Lost to follow } \\
\text { up per } \\
\text { outcome }(\%)\end{array}$ & $\begin{array}{l}\text { Follow } \\
\text { up in } \\
\text { months }\end{array}$ \\
\hline \multicolumn{8}{|c|}{ Laparoscopy versus laparotomy } \\
\hline \multirow{2}{*}{$\begin{array}{l}\text { Lundorff } \\
(1991+1992)\end{array}$} & \multirow{2}{*}{$\begin{array}{l}1987- \\
1989\end{array}$} & \multirow{2}{*}{$\begin{array}{l}\text { Patients with ectopic } \\
\text { pregnancy }\end{array}$} & Laparoscopy & 48 & \multirow{2}{*}{ Pregnancy } & \multirow{2}{*}{$18 / 105(17.1)$} & \multirow{2}{*}{$1-36$} \\
\hline & & & Laparotomy & 57 & & & \\
\hline \multirow{2}{*}{$\begin{array}{l}\text { Takahashi } \\
(2007)\end{array}$} & \multirow[t]{2}{*}{ NR } & \multirow[t]{2}{*}{$\begin{array}{l}\text { Polycystic ovarian } \\
\text { syndrome }\end{array}$} & Laparoscopy & 39 & $\begin{array}{l}\text { Second } \\
\text { look }\end{array}$ & $0 / 76(0)$ & 1 week \\
\hline & & & Laparotomy & 37 & Pregnancy & $0 / 76(0)$ & 12 \\
\hline \multicolumn{8}{|c|}{ Peritoneal closure versus no peritoneal closure } \\
\hline \multirow{2}{*}{$\begin{array}{l}\text { Kadanali } \\
\text { (1996) }\end{array}$} & \multirow{2}{*}{$\begin{array}{l}1992- \\
1995\end{array}$} & \multirow{2}{*}{$\begin{array}{l}\text { Lymphadenectomy } \\
\text { in ovarian cancer }\end{array}$} & $\begin{array}{l}\text { Peritoneal } \\
\text { closure }\end{array}$ & 50 & \multirow{2}{*}{$\begin{array}{l}\text { Second } \\
\text { look }\end{array}$} & \multirow[t]{2}{*}{$0 / 102(0)$} & \multirow[t]{2}{*}{ NR } \\
\hline & & & $\begin{array}{l}\text { No peritoneal } \\
\text { closure }\end{array}$ & 52 & & & \\
\hline \multirow{2}{*}{$\begin{array}{l}\text { Franchi } \\
\text { (1997) }\end{array}$} & \multirow{2}{*}{$\begin{array}{l}1991- \\
1995\end{array}$} & \multirow{2}{*}{$\begin{array}{l}\text { Hysterectomy and } \\
\text { pelvic node } \\
\text { dissection }\end{array}$} & $\begin{array}{l}\text { Peritoneal } \\
\text { closure }\end{array}$ & 59 & \multirow{2}{*}{$\begin{array}{l}\text { ASBO- } \\
\text { reoperation }\end{array}$} & \multirow[t]{2}{*}{$0 / 120(0)$} & \multirow[t]{2}{*}{$11-72$} \\
\hline & & & $\begin{array}{l}\text { No peritoneal } \\
\text { closure }\end{array}$ & 61 & & & \\
\hline \multirow{2}{*}{$\begin{array}{l}\text { Malvasi } \\
(2009)\end{array}$} & \multirow{2}{*}{$\begin{array}{l}2003- \\
2007\end{array}$} & \multirow[t]{2}{*}{ Caesarean section } & $\begin{array}{l}\text { Peritoneal } \\
\text { closure }\end{array}$ & 54 & \multirow{2}{*}{$\begin{array}{l}\text { Second } \\
\text { look }\end{array}$} & \multirow[t]{2}{*}{$0 / 112(0)$} & \multirow[t]{2}{*}{ NR } \\
\hline & & & $\begin{array}{l}\text { No peritoneal } \\
\text { closure }\end{array}$ & 58 & & & \\
\hline
\end{tabular}




\begin{tabular}{|c|c|c|c|c|c|c|c|}
\hline Study & Period & Patients & Interventions & $\mathbf{N}$ & Outcomes & $\begin{array}{l}\text { Lost to follow } \\
\text { up per } \\
\text { outcome }(\%)\end{array}$ & $\begin{array}{l}\text { Follow } \\
\text { up in } \\
\text { months }\end{array}$ \\
\hline \multicolumn{8}{|c|}{ Laser versus electrocautery } \\
\hline \multirow{2}{*}{$\begin{array}{l}\text { Gürgan } \\
\text { (1991) }\end{array}$} & \multirow[t]{2}{*}{ NR } & \multirow{2}{*}{$\begin{array}{l}\text { Poly cystic ovarian } \\
\text { syndrome }\end{array}$} & Laser & 10 & $\begin{array}{l}\text { Second } \\
\text { look }\end{array}$ & $0 / 17(0)$ & $\begin{array}{l}3-4 \\
\text { weeks }\end{array}$ \\
\hline & & & $\begin{array}{l}\text { Electrocautery } \\
\text { (unipolar) }\end{array}$ & 7 & Pregnancy & $0 / 17(0)$ & 6 \\
\hline \multirow[b]{2}{*}{$\begin{array}{l}\text { Tulandi } \\
\text { (1986) }\end{array}$} & \multirow{2}{*}{ NR } & \multirow{2}{*}{$\begin{array}{l}\text { Periadnexal } \\
\text { adhesions }\end{array}$} & Laser & 30 & \multirow{2}{*}{ Pregnancy } & \multirow{2}{*}{$0 / 63(0)$} & \multirow{2}{*}{ NR } \\
\hline & & & $\begin{array}{l}\text { Electrocautery } \\
\text { (unipolar) }\end{array}$ & 33 & & & \\
\hline \multicolumn{8}{|c|}{ Sutures versus electrocautery } \\
\hline \multirow{2}{*}{$\begin{array}{l}\text { Pellicano } \\
(2008)\end{array}$} & \multirow{2}{*}{$\begin{array}{l}2004- \\
2005\end{array}$} & \multirow{2}{*}{$\begin{array}{l}\text { Ovarian } \\
\text { endometrioma }\end{array}$} & Suturing & 16 & \multirow{2}{*}{$\begin{array}{l}\text { Second } \\
\text { look }\end{array}$} & \multirow{2}{*}{$5 / 32(15.6)$} & \multirow{2}{*}{$\begin{array}{l}60-90 \\
\text { days }\end{array}$} \\
\hline & & & $\begin{array}{l}\text { Electrocautery } \\
\text { (bipolar) }\end{array}$ & 16 & & & \\
\hline \multicolumn{8}{|c|}{ Second look surgery versus no second look surgery } \\
\hline \multirow{2}{*}{$\begin{array}{l}\text { Alborzi } \\
(2003)\end{array}$} & \multirow{2}{*}{ NR } & \multirow{2}{*}{ Adnexal adhesions } & Second look & 46 & \multirow{2}{*}{ Pregnancy } & \multirow{2}{*}{$0 / 90(0)$} & \multirow{2}{*}{12} \\
\hline & & & $\begin{array}{l}\text { No second } \\
\text { look }\end{array}$ & 44 & & & \\
\hline \multirow[b]{2}{*}{$\begin{array}{l}\text { Gürgan } \\
\text { (1992) }\end{array}$} & \multirow{2}{*}{ NR } & \multirow{2}{*}{$\begin{array}{l}\text { Poly Cystic ovarian } \\
\text { syndrome }\end{array}$} & Second look & 19 & \multirow{2}{*}{ Pregnancy } & \multirow{2}{*}{$0 / 39(0)$} & \multirow{2}{*}{6} \\
\hline & & & $\begin{array}{l}\text { No second } \\
\text { look }\end{array}$ & 20 & & & \\
\hline \multicolumn{8}{|c|}{ Various techniques in laparoscopic surgery for polycystic ovarian syndrome } \\
\hline \multirow{2}{*}{$\begin{array}{l}\text { Sharma } \\
(2006)\end{array}$} & \multirow[t]{2}{*}{ NR } & Poly cystic ovarian & $\begin{array}{l}\text { Unipolar } \\
\text { electrocautery }\end{array}$ & 10 & Pregnancy & $0 / 20(0)$ & NR \\
\hline & & & $\begin{array}{l}\text { Bipolar } \\
\text { electrocautery }\end{array}$ & 10 & & & \\
\hline Mercorio & 2002- & Poly cystic ovarian & 12 punctures & $96^{\mathrm{a}}$ & Second & $12 / 182^{\mathrm{a}}(6,3)$ & $4-9$ \\
\hline$(2008)$ & 2006 & syndrome & 6 punctures & $96^{\mathrm{a}}$ & look & & weeks \\
\hline & $2005-$ & Poly cystic ovarian & Bilateral & 22 & Pregnancy & $0 / 44$ & 12 \\
\hline Roy (2009) & 2007 & syndrome & Unilateral & 22 & & & \\
\hline Miscellaneo & & & & & & & \\
\hline Pellicano & $2001-$ & Laparoscopic & $\begin{array}{l}\text { Subserous } \\
\text { sutures }\end{array}$ & 18 & Pregnancy & $0 / 36$ & 12 \\
\hline$(2005)$ & & Miny & $\begin{array}{l}\text { Figure-eigth } \\
\text { sutures }\end{array}$ & 18 & & & \\
\hline
\end{tabular}

NR: not reported; ${ }^{a}$ randomization unit is ovary.

Table 4: Characteristics of studies included in qualitative assessment.

\begin{tabular}{|c|c|c|c|c|c|c|c|}
\hline Study & Period & Patients & Interventions & $\mathbf{N}$ & Outcomes & $\begin{array}{l}\text { Lost to follow } \\
\text { up per } \\
\text { outcome }(\%)\end{array}$ & $\begin{array}{l}\text { Follow } \\
\text { up in } \\
\text { months }\end{array}$ \\
\hline \multicolumn{8}{|c|}{ Laparoscopy versus laparotomy } \\
\hline \multirow{2}{*}{$\begin{array}{l}\text { Lundorff } \\
(1991-1993)\end{array}$} & \multirow{2}{*}{$\begin{array}{l}1987- \\
1989\end{array}$} & \multirow[t]{2}{*}{$\begin{array}{l}\text { Patients with } \\
\text { ectopic pregnancy }\end{array}$} & Laparoscopy & 48 & Second look & $32 / 105(27.8)$ & $\begin{array}{l}12 \\
\text { weeks }\end{array}$ \\
\hline & & & Laparotomy & 57 & Pregnancy & $18 / 105(17.1)$ & $1-36$ \\
\hline \multirow{2}{*}{$\begin{array}{l}\text { Stocchi } \\
(2008)\end{array}$} & \multirow[b]{2}{*}{ NR } & \multirow{2}{*}{$\begin{array}{l}\text { Ileocolic resection } \\
\text { for Crohn's disease }\end{array}$} & Laparoscopy & 27 & \multirow{2}{*}{$\begin{array}{l}\text { ASBO- } \\
\text { reoperation }\end{array}$} & $0 / 27(0)$ & $120 \pm 38^{\mathrm{a}}$ \\
\hline & & & Laparotomy & 29 & & $0 / 29(0)$ & $132 \pm 17^{\mathrm{a}}$ \\
\hline \multirow{2}{*}{$\begin{array}{l}\text { Taylor } \\
(2010)\end{array}$} & \multirow{2}{*}{$\begin{array}{l}1996- \\
2002\end{array}$} & \multirow{2}{*}{ Colorectal cancer } & Laparoscopy & 526 & \multirow{2}{*}{$\begin{array}{l}\text { ASBO- } \\
\text { reoperation }\end{array}$} & $246 / 526(46.8)$ & \multirow[t]{2}{*}{36} \\
\hline & & & Laparotomy & 268 & & $131 / 268(48.9)$ & \\
\hline \multicolumn{8}{|c|}{ Peritoneal closure versus no peritoneal closure } \\
\hline $\begin{array}{l}\text { Kapustian } \\
\text { (2011) }\end{array}$ & $\begin{array}{l}2004- \\
2007\end{array}$ & Caesarean section & $\begin{array}{l}\text { Peritoneal } \\
\text { closure }\end{array}$ & 47 & Second look & $436 / 533(81.8)$ & NR \\
\hline
\end{tabular}




\begin{tabular}{|c|c|c|c|c|c|c|c|}
\hline Study & Period & Patients & Interventions & $\mathbf{N}$ & Outcomes & $\begin{array}{l}\text { Lost to follow } \\
\text { up per } \\
\text { outcome }(\%)\end{array}$ & $\begin{array}{l}\text { Follow } \\
\text { up in } \\
\text { months }\end{array}$ \\
\hline & & & $\begin{array}{l}\text { No peritoneal } \\
\text { closure }\end{array}$ & 50 & & & \\
\hline \multirow{2}{*}{$\begin{array}{l}\text { Komoto } \\
(2006)\end{array}$} & \multirow{2}{*}{$\begin{array}{l}1995- \\
2000\end{array}$} & \multirow[t]{2}{*}{ Caesarean section } & $\begin{array}{l}\text { Peritoneal } \\
\text { closure }\end{array}$ & 70 & \multirow{2}{*}{ Second look } & \multirow[t]{2}{*}{$74 / 124(59.7)$} & \multirow[t]{2}{*}{ NR } \\
\hline & & & $\begin{array}{l}\text { No peritoneal } \\
\text { closure }\end{array}$ & 54 & & & \\
\hline \multirow{2}{*}{$\begin{array}{l}\text { Weerawetwat } \\
(2004)\end{array}$} & \multirow{2}{*}{$\begin{array}{l}1987- \\
1991\end{array}$} & \multirow[t]{2}{*}{ Caesarean section } & $\begin{array}{l}\text { Peritoneal } \\
\text { closure }\end{array}$ & 240 & \multirow{2}{*}{ Second look } & \multirow[t]{2}{*}{$295 / 360(81.9)$} & \multirow[t]{2}{*}{ NR } \\
\hline & & & $\begin{array}{l}\text { No peritoneal } \\
\text { closure }\end{array}$ & 120 & & & \\
\hline \multirow{2}{*}{$\begin{array}{l}\text { Zareian } \\
(2006)\end{array}$} & \multirow{2}{*}{$\begin{array}{l}1999- \\
2004\end{array}$} & \multirow[t]{2}{*}{ Caesarean section } & $\begin{array}{l}\text { Peritoneal } \\
\text { closure }\end{array}$ & 24 & \multirow{2}{*}{ Second look } & \multirow[t]{2}{*}{$14 / 45(31.1)$} & \multirow[t]{2}{*}{ NR } \\
\hline & & & $\begin{array}{l}\text { No peritoneal } \\
\text { closure }\end{array}$ & 21 & & & \\
\hline \multicolumn{8}{|c|}{ Techniques in cesarean section } \\
\hline \multirow{2}{*}{$\begin{array}{l}\text { Nabhan } \\
(2008)\end{array}$} & \multirow{2}{*}{$\begin{array}{l}2002- \\
2007\end{array}$} & \multirow[t]{2}{*}{ Caesarean section } & $\begin{array}{l}\text { Pfannenstiel- } \\
\text { Kerr }\end{array}$ & 300 & \multirow{2}{*}{ Second look } & \multirow[t]{2}{*}{$476 / 600(79.3)$} & \multirow[t]{2}{*}{ NR } \\
\hline & & & $\begin{array}{l}\text { Joel-Cohen- } \\
\text { Stark }\end{array}$ & 300 & & & \\
\hline \multicolumn{8}{|c|}{ Suturing versus no suturing following salpingotomy } \\
\hline \multirow{2}{*}{$\begin{array}{l}\text { Fujishita } \\
(2004)\end{array}$} & \multirow{2}{*}{$\begin{array}{l}1996- \\
2002\end{array}$} & \multirow{2}{*}{$\begin{array}{l}\text { Salpingotomy for } \\
\text { ectopic pregnancy }\end{array}$} & Sutures & 32 & \multirow{2}{*}{ Pregnancy } & \multirow{2}{*}{$43 / 75(57.3)$} & \multirow{2}{*}{$6-65$} \\
\hline & & & No sutures & 43 & & & \\
\hline \multirow{2}{*}{$\begin{array}{l}\text { Tulandi } \\
\text { (1991) }\end{array}$} & \multirow{2}{*}{ NR } & \multirow{2}{*}{$\begin{array}{l}\text { Salpingotomy for } \\
\text { ectopic pregnancy }\end{array}$} & Sutures & 19 & \multirow{2}{*}{ Second look } & $8 / 19(42.1)$ & 24 \\
\hline & & & No sutures & 15 & & $8 / 15(53.3)$ & \\
\hline Taskin & NR & $\begin{array}{l}\text { Poly cystic ovarian } \\
\text { syndrome }\end{array}$ & $\begin{array}{l}\text { Microlaparosco } \\
\text { py }\end{array}$ & 9 & Second look & $? / 9$ & $\begin{array}{l}2-3 \\
\text { weeks }\end{array}$ \\
\hline & & & Laparoscopy & 9 & & $? / 9$ & \\
\hline
\end{tabular}

Randomization unit is ovary; NR: not reported; median (range); ${ }^{\mathrm{a}}$ mean $\pm \mathrm{SD}$.

\section{DISCUSSION}

\section{Summary of evidence}

Surgical techniques aiming to reduce adhesion formation included a large variety of technical aspects. None of the different techniques or approaches evidently showed a reduction of the main adhesion-related complications ASBO (adhesive small bowel obstruction) and infertility. The incidence of ASBO, established by reoperation was not significantly different in any comparison. The clinical suspicion of ASBO was lower following laparoscopy compared with open surgery in one study. The incidence of adhesions was lower following laparoscopy than laparotomy and when the peritoneum was left open compared with peritoneal closure. However, the evidence for a lower incidence of adhesions was limited to a single small RCT and conflicting results were found in the qualitative assessment of lower quality studies. The pregnancy rate was significantly higher in one study after subserous fixation of sutures compared with standard sutures in a small low-quality RCT.

\section{Strengths and limitations of the review}

The failure to demonstrate an effect on a relevant clinical end-point such as ASBO and pregnancy in this metaanalysis has several causes. Particularly in gynaecological studies, a substantial portion of patients were lost to follow up decreasing the number of evaluable patients. One single adhesive band may still cause a bowel obstruction.

The present study was the first systematic review and meta-analysis of the impact of different surgical techniques on adhesion formation. The available evidence was predominantly from surgery of gynaecologic origin, particularly related to fertility by Metwally et al 2006 and Ahmad et al 2008). ${ }^{9,10}$ This type of surgery was often chosen in adhesion prevention research, because of the historical awareness of the adhesion problem within the European and the American fertility societies and because the surgery included a second-look procedure and prevention of local adhesion reformation corresponded with clinical success by Zerega 1993, Marana et al 1995 and Nappi et al 2007.4,11,12 
A large number of studies had difficulty in achieving a complete follow up. As many as nine studies were excluded from meta-analysis because of an inadequate follow up. Especially, the repeat caesarean section model seems prone to high numbers of patients lost to follow up, a study done by Weerawetwat et al 2004, Komoto et al 2006, Zareian et al 2006, Nabhan et al 2008 and Kapustian et al 2011. ${ }^{13-17}$ Further, the choice of a repeat caesarean section as a second-look procedure to study peritoneal closure bears the risk of selection bias towards patients with fewer adhesions because they had a higher chance of becoming pregnant again. Such study design also led to a large variation in the follow up period, as the timing of the next pregnancy and the need for another caesarean section were unpredictable.

Unpublished data from a large RCT revealed that every 30 min of adhesiolysis was correlated to an increase in hospital stay with 1 day, study done by Fazio et al $2006 .{ }^{18}$

\section{Comparison with previous research}

The reduction in adhesion extent and severity by limited electrocoagulation, subserous suture fixation and nonclosure of the peritoneum emphasized the importance of limiting peritoneal ischaemia and foreign body material in surgery, study done by Kadanali et al 1996 and Pellicano et al 2003. ${ }^{6,19}$ Contrastingly, current guidelines suggested that non-closure might be more favourable in terms of short-term complications, recovery and adhesion formation (Royal college of obstetricians and gynecologists, 2002; National institute of clinical excellence, 2004). A Cochrane review studying short-term complications and recovery after caesarean section found no difference between closure and non-closure of the peritoneum, a study done by Bamigboye et al 2009. ${ }^{20} \mathrm{~A}$ recent large RCT of caesarean sections also demonstrated no difference in short-term complications. Long-term follow up results were still awaited by Caesar study collaborative group, 2010. ${ }^{21}$ Six RCTs addressing peritoneal closure in general surgery and following hysterectomy have demonstrated a similar incidence of incisional hernia after closure or non-closure of the peritoneum, studies done by Ellis et al 1977; McFadden et al 1983; Gilbert et al 1987; Hugh et al 1990; Than et al 1994 and Lipscomb et al 1996). ${ }^{22-27}$ Summarizing the results of suturing or not suturing the peritoneum in caesarean section, both techniques seemed acceptable considering short-term complications but non-closure might decreased incidence of adhesions. The question was why adhesions were not more effectively prevented despite the strong concept of minimal invasive surgery inducing less tissue damage and thus a lower risk of adhesion formation. A number of factors might explain the lack of difference between laparoscopic and open surgery. First, an abdominal incision was often needed after a laparoscopic procedure to extract the specimen by an open approach. Secondly, the extent of serosal wound surfaces was comparable between open and laparoscopic procedures. Thirdly, the $\mathrm{CO}_{2}$ pneumoperitoneum, the higher intra-abdominal pressure and the light of the laparoscope being associated with peritoneal ischaemia, decreased fibrinolysis and increased adhesion formation, studies done by Binda et al 2003 and Brokelman et al 2006). ${ }^{28,29}$ Fourth, the pneumoperitoneum potentially injured the whole peritoneal surface inducing adhesion formation at remote areas. More meticulous dissection and haemostasis, no retraction of the abdominal wall and no use of gauzes in the peritoneal cavity in laparoscopic surgery counterbalance the drawbacks mentioned.

\section{Implications for future research}

The poor quality of RCT's and the limited number of eligible patients illustrate the main difficulty in clinical adhesion research, the execution of a planned second-look operation, which was the gold standard for assessment of the incidence and severity of adhesions. The number of planned second procedures had declined over recent years in both female patients who underwent planned secondlook laparoscopy following fertility surgery. The declining number of planned second procedures was a challenge for future research in adhesion prevention. Visceral slide and cine-MRI were non-invasive adhesion detection techniques that have the potential to replace a second-look operation. Cine-MRI especially holds promise identifying both adhesions to the abdominal wall and between abdominal viscera, studies done by Lienemann et al 2000; Kirchhoff et al 2010. ${ }^{31,32}$

\section{Implications for clinical practice}

None of the different techniques had a major impact on adhesion-related complications. This meta-analysis provided little evidence that less invasive techniques, less foreign body material and less ischaemic injury reduced the extent and severity of adhesions in humans. The total prevention of adhesion formation was the only means to prevent an adhesion-related complication. It was not expected that optimal surgical technique alone will achieve this goal, based on the inevitable peritoneal injury inflicted by any type of surgery. As a consequence, there continued to be a need for anti-adhesion barriers and agents in open and laparoscopic surgery, studies done by Diamond 1996; Zerega et al 2002; Fazio et al 2006 and Metwally et al 2006..$^{9,18,33,34}$

\section{CONCLUSION}

Adhesion development is a significant, yet poorly understood cause of morbidity in post-operative patients. To date, it remains unknown exactly why adhesions form more frequently in certain tissues and/or patients, or at specific locations, as opposed to others. This review contributes to the growing knowledge pool by elucidating factors that potentially predispose to the development of adhesions. By identifying those factors shown to directly increase risk (genetic polymorphisms, estrogen exposure and endometriosis) in addition to those that might do so indirectly by way of altering the coagulation/fibrinolytic 
profile in such a way that increases fibrosis (genetic polymorphisms, diabetes mellitus, metabolic syndrome, hyperglycemia, obesity, depression, binge alcohol consumption, anti-Parkinsonian medications, oral hormone therapy, pregnancy and cancer), this review can be a useful tool for surgeons to identify high-risk patients who might benefit from anti-adhesion agents. Furthermore, this review serves as a useful catalyst for inspiring future areas of investigation. Further research is necessary to understand the mechanisms that underlie the association of the factors identified in this review with adhesion formation. Future research should also investigate whether there exists a direct link between adhesion formation and any of the factors we have identified as potentially doing so indirectly by increasing fibrosis. This information will be crucial in the creation of adequate preventative and treatment strategies.

\section{Funding: No funding sources}

Conflict of interest: None declared

Ethical approval: Not required

\section{REFERENCES}

1. Milingos S, Kallipolitis G, Loutradis D, Liapi A, Mavrommatis K, Drakakis $\mathrm{P}$, et al. Adhesions: laparoscopic surgery versus laparotomy. Ann N Y Acad Sci.2000;900:272-85.

2. Swank DJ, Erp WFV, Driel OJRV, Hop WC, Bonjer HJ, Jeekel H. A prospective analysis of predictive factors on the results of laparoscopic adhesiolysis in patients with chronic abdominal pain. Lancet. 2003;361(9365):1247-51.

3. Broek RPT, Schreinemacher MH, Jilesen AP, Bleichrodt RP, Goor HV. Enterotomy risk in abdominal wall repair: a prospective study. Ann Surg. 2012;256(2):280-7.

4. Zerega GSD. The cause and prevention of postsurgical adhesions: a contemporary update. Prog Clin Biol Res. 1993;381:1-18.

5. Taskin O, Sadik S, Onoglu A, Gokdeniz R, Yilmaz I, Burak F, et al. Adhesion formation after microlaparoscopic and laparoscopic ovarian coagulation for polycystic ovary disease. J Am Assoc Gynecol Laparosc. 1999;6:159-63.

6. Pellicano M, Bramante S, Cirillo D, Palomba S, Bifulco G, Zullo F, et al. Effectiveness of auto cross linked hyaluronic acid gel after laparoscopic myomectomy in infertile patients: a prospective, randomized, controlled study. Fertil Steril. 2003;80(2):441-4.

7. Diamond MP, Wexner SD, diZereg GS, Korell M, Zmora O, Goor HV, et al. Adhesion prevention and reduction: current status and future recommendations of a multinational interdisciplinary consensus conference. Surg Innov. 2010;17:183-8.

8. Lundorff $\mathrm{P}$, Hahlin M, Kallfelt B, Thorburn J, Lindblom B. Adhesion formation after laparoscopic surgery in tubal pregnancy: a randomized trial versus laparotomy. Fertil Steril. 1991;55(5):911-5.
9. Metwally M, Watson A, Lilford R, Vandekerckhove P. Fluid and pharmacological agents for adhesion prevention after gynaecological surgery. Cochrane Database Syst Rev. 2006;2:001298.

10. Ahmad G, Duffy JM, Farquhar C, Vail A, Vandekerckhove P, Watson A, et al. Barrier agents for adhesion prevention after gynaecological surgery. Cochrane Database Syst Rev. 2008;2:000475.

11. Marana R, Rizzi M, Muzii L, Catalano GF, Caruana $\mathrm{P}$, Mancuso S. Correlation between the American Fertility Society classifications of adnexal adhesions and distal tubal occlusion, salpingoscopy, and reproductive outcome in tubal surgery. Fertil Steril. 1995;64:924-9.

12. Nappi C, DiSpiezio SA, Greco E, Guida M, Bettocchi $S$, Bifulco G. Prevention of adhesions in gynaecological endoscopy. Hum Reprod Update. 2007;13(4):379-94.

13. Weerawetwat W, Buranawanich S, Kanawong M. Closure vs non-closure of the visceral and parietal peritoneum at cesarean delivery: 16 year study. J Med Assoc Thai. 2004;87(9):1007-11.

14. Komoto Y, Shimoya K, Shimizu T, Kimura T, Hayashi S, Temma-Asano K, et al. Prospective study of non-closure or closure of the peritoneum at cesarean delivery in 124 women: impact of prior peritoneal closure at primary cesarean on the interval time between first cesarean section and the next pregnancy and significant adhesion at second caesarean. J Obstet Gynaecol Res. 2006;32(4):396402.

15. Zareian Z, Zareian P. Non-closure versus closure of peritoneum during cesarean section: a randomized study. Eur J Obstet Gynecol Reprod Biol. 2006;128(12):267-9.

16. Nabhan AF. Long-term outcomes of two different surgical techniques for caesarean. Int $\mathbf{J}$ Gynaecol Obstet. 2008;100(1):69-75.

17. Kapustian V, Anteby EY, Gdalevich M, Shenhav S, Lavie O, Gemer O. Effect of closure versus nonclosure of peritoneum at cesarean section on adhesions: a prospective randomized study. Am J Obstet Gynecol. 2012;206(1):56.

18. Fazio VW, Cohen Z, Fleshman JW, vanGoor H, Bauer JJ, Wolff BG, et al. Reduction in adhesive smallbowel obstruction by Seprafilm adhesion barrier after intestinal resection. Dis Colon Rectum. 2006;49(1):111.

19. Kadanali S, Erten O, Kucukozkan T. Pelvic and periaortic pertioneal closure or non-closure at lymphadenectomy in ovarian cancer: effects on morbidity and adhesion formation. Eur J Surg Oncol. 1996;22(3):282-5.

20. Bamigboye AA, Hofmeyr GJ. Local anaesthetic wound infiltration and abdominal nerves block during caesarean section for postoperative pain relief. Cochrane Database Syst Rev. 2009;3:006954.

21. CAESAR study collaborative group. Caesarean section surgical techniques: a randomised factorial trial (CAESAR). BJOG. 2010;117(11):1366-76. 
22. Ellis H, Moran BJ, Thompson JN, Parker MC, Wilson MS, Menzies D, et al. Adhesion-related hospital readmissions after abdominal and pelvic surgery: a retrospective COHORT study. Lancet. 1999;353(9163):1476-80.

23. McFadden PM, Peacock EE. Preperitoneal abdominal wound repair: incidence of dehiscence. Am J Surg. $1983 ; 145(2): 213-4$

24. Gilbert JM, Ellis H, Foweraker S. Peritoneal closure after lateral paramedian incision. $\mathrm{Br} \mathrm{J}$ Surg. 1987;74(2):113-5.

25. Hugh TB, Nankivell C, Meagher AP, Li B. Is closure of the peritoneal layer necessary in the repair of midline surgical abdominal wounds? World J Surg. 1990;14(2):231-3.

26. Than GN, Arany AA, Schunk E, Vizer M, Krommer KF. Closure or non-closure of visceral peritoneums after abdominal hysterectomies and Wertheim-Meigs radical abdominal hysterectomies. Acta Chir Hung. 1994;34:79-86.

27. Lipscomb GH, Ling FW, Stovall TG, Summitt RL. Peritoneal closure at vaginal hysterectomy: a reassessment. Obstet Gynecol. 1996;87(1):40-3.

28. Binda MM, Molinas CR, Koninckx PR. Reactive oxygen species and adhesion formation: clinical implications in adhesion prevention. Hum Reprod. 2003;18(12):2503-7.

29. Brokelman WJ, Holmdahl L, Bergstrom M, Falk P, Klinkenbijl JH, Reijnen MM. Peritoneal fibrinolytic response to various aspects of laparoscopic surgery: a randomized trial. J Surg Res. 2006;136:309-13.

30. Binnebosel M, Klink CD, Grommes J, Jansen M, Neumann UP, Junge K. Influence of small intestinal serosal defect closure on leakage rate and adhesion formation: a pilot study using rabbit models. Langenbecks Arch Surg. 2011;396:133-7.

31. Kirchhoff S, Ladurner R, Kirchhoff C, Mussack T, Reiser MF, Lienemann A. Detection of recurrent hernia and intraabdominal adhesions following incisional hernia repair: a functional cine MRI-study. Abdom Imaging. 2010;35(2):224-31.

32. Lienemann A, Sprenger D, Steitz HO, Korell M, Reiser M. Detection and mapping of intraabdominal adhesions by using functional cine MR imaging: preliminary results. Radiology. 2000;217(2):421-5.

33. Diamond MP. Reduction of adhesions after uterine myomectomy by Seprafilm membrane (HAL-F): a blinded, prospective, randomized, multicenter clinical study. Seprafilm Adhesion study group. Fertil Steril. 1996;66(6):904-10.

34. diZerega GS, Verco SJ, Young P, Kettel M, Kobak W, Martin D, et al. A randomized, controlled pilot study of the safety and efficacy of $4 \%$ icodextrin solution in the reduction of adhesions following laparoscopic gynaecological surgery. Hum Reprod. 2002;17(4):1031-8.

35. Chen MD, Teigen GA, Reynolds HT, Johnson PR, Fowler JM. Laparoscopy versus laparotomy: an evaluation of adhesion formation after pelvic and paraaortic lymphadenectomy in a porcine model. Am J Obstet Gynecol. 1998;178(3):499-503.

36. diZerega GS. The cause and prevention of postsurgical adhesions: a contemporary update. Prog Clin Biol Res. 1993;381:1-18.

37. Duffy DM, diZerega GS. Is peritoneal closure necessary? Obstet Gynecol Surv. 1994;49(12):81722.

38. Duron JJ. Postoperative intraperitoneal adhesion pathophysiology. Colorectal Dis. 2007;9(2):14-24.

39. Ellis H, Heddle R. Does the peritoneum need to be closed at laparotomy? Br J Surg. 1977;64(10):733-6.

40. Eshuis EJ, Slors JF, Stokkers PC, Sprangers MA, Ubbink DT, Cuesta MA, et al. Long-term outcomes following laparoscopically assisted versus open ileocolic resection for Crohn's disease. Br J Surg. 2010;97:563-8.

41. Foster NM, McGory ML, Zingmond DS, Ko CY. Small bowel obstruction: a population-based appraisal. J Am Coll Surg. 2006;203(2):170-6.

42. Franchi M, Ghezzi F, Zanaboni F, Scarabelli C, Beretta P, Donadello N. Nonclosure of peritoneum at radical abdominal hysterectomy and pelvic node dissection: a randomized study. Obstet Gynecol. 1997;90:622-7.

43. Fujishita A, Masuzaki H, Khan KN, Kitajima M, Hiraki K, Ishimaru T. Laparoscopic salpingotomy for tubal pregnancy: comparison of linear salpingotomy with and without suturing Hum Reprod 2004;19(5):1195-200.

44. Godinjak Z, Idrizbegovic E. Should diagnostic hysteroscopy be a routine procedure during diagnostic laparoscopy in infertile women? Bosn J Basic Med Sci. 2008;8(1):44-7.

45. Gurgan T, Kisnisci H, Yarali H, Develioglu O, Zeyneloglu H, Aksu T. Evaluation of adhesion formation after laparoscopic treatment of polycystic ovarian disease. Fertil Steril. 1991;56:1176-8.

46. Gurgan T, Urman B, Aksu T, Yarali H, Develioglu O, Kisnisci HA. The effect of short-interval laparoscopic lysis of adhesions on pregnancy rates following $\mathrm{Nd}$ YAG laser photocoagulation of polycystic ovaries. Obstet Gynecol. 1992;80(1):45-7.

47. Hammoud AO, Gibson M, Stanford J, White G, Carrell DT, Peterson M. In vitro fertilization availability and utilization in the United States: a study of demographic, social, and economic factors. Fertil Steril. 2009;91(5):1630-5.

48. Hiki N, Shimizu N, Yamaguchi H, Imamura K, Kami $\mathrm{K}$, Kubota K, et al. Manipulation of the small intestine as a cause of the increased inflammatory response after open compared with laparoscopic surgery. Br J Surg. 2006;93:195-204.

49. Ivarsson ML, Bergstrom M, Eriksson E, Risberg B, Holmdahl L. Tissue markers as predictors of postoperative adhesions. $\mathrm{Br} \quad \mathrm{J}$ Surg. 1998;85(11):1549-54.

50. Kapur ML, Daneswar A, Chopra P. Evaluation of peritoneal closure at laparotomy. Am J Surg. 1979;137(5):650-2. 
51. Krahenbuhl L, Schafer M, Kuzinkovas V, Renzulli P, Baer HU, Buchler MW. Experimental study of adhesion formation in open and laparoscopic fundoplication. Br J Surg. 1998;85:826-30.

52. Kyzer S, Bayer I, Turani H, Chaimoff C. The influence of peritoneal closure on formation of intraperitoneal adhesions: an experimental study. Int J Tissue React. 1986;8(5):355-9.

53. Levrant SG, Bieber EJ, Barnes RB. Anterior abdominal wall adhesions after laparotomy or laparoscopy. J Am Assoc Gynecol Laparosc. 1997;4(3):353-6.

54. Lim R, Morrill JM, Lynch RC, Reed KL, Gower AC, Leeman SE, et al. Practical limitations of bioresorbable membranes in the prevention of intraabdominal adhesions. J Gastrointest Surg. 2009;13(1):35-41.

55. Lower AM, Hawthorn RJ, Ellis H, O'Brien F, Buchan S, Crowe AM. The impact of adhesions on hospital readmissions over ten years after 8849 open gynaecological operations: an assessment from the surgical and clinical adhesions research study. BJOG. 2000;107(7):855-62.

56. Lower AM, Hawthorn RJ, Clark D, Boyd JH, Finlayson AR, Knight AD, et al. Adhesion-related readmissions following gynaecological laparoscopy or laparotomy in Scotland: an epidemiological study of 24046 patients. Hum Reprod. 2004;19(8):1877-85.

57. Luijendijk RW, Wauters CC, Hop WC, Duron JJ, Pailler JL, Camprodon BR, et al. Foreign material in postoperative adhesions. Ann Surg. 1996;223(3):2428.

58. Lundorff $\mathrm{P}$, Thorburn J, Lindblom B. Fertility outcome after conservative surgical treatment of ectopic pregnancy evaluated in a randomized trial. Fertil Steril. 1992;57(5):998-1002.

59. Lyell DJ, Caughey AB, Hu E, Daniels K. Peritoneal closure at primary cesarean delivery and adhesions. Obstet Gynecol. 2005;106(2):275-80.

60. Malvasi A, Tinelli A, Farine D, Rahimi S, Cavallotti C, Vergara D, et al. Effects of visceral peritoneal closure on scar formation at cesarean delivery. Int $\mathrm{J}$ Gynaecol Obstet. 2009;105:131-5.

61. Marana R, Luciano AA, Muzii L, Marendino VE, Mancuso S. Laparoscopy versus laparotomy for ovarian conservative surgery: a randomized trial in the rabbit model. Am J Obstet Gynecol. 1994;171:861-4.

62. Mercorio F, Mercorio A, DiSpiezio SA, Barba GV, Pellicano M, Nappi C. Evaluation of ovarian adhesion formation after laparoscopic ovarian drilling by second-look minilaparoscopy. Fertil Steril. 2008;89(5):1229-33.

63. Mowat C, Cole A, Windsor A, Ahmad T, Arnott I, Driscoll R, et al. Guidelines for the management of inflammatory bowel disease in adults. Gut. 2011;60(5):571-607.

64. Nakagawa K, Ohgi S, Horikawa T, Kojima R, Ito M, Saito H. Laparoscopy should be strongly considered for women with unexplained infertility. J Obstet Gynaecol Res. 2007;33(5):665-70.
65. National Institute of Clinical Excellence. Caesarean section. Clinical guidelines 2004, guideline no. 13. London: RCOG Press; 2011.

66. Ng SS, Leung KL, Lee JF, Yiu RY, Li JC, Hon SS. Long-term morbidity and oncologic outcomes of laparoscopic-assisted anterior resection for upper rectal cancer: ten-year results of a prospective, randomized trial. Dis Colon Rectum. 2009;52(4):55866.

67. Nieuwenhuijzen M, Reijnen MM, Kuijpers JH, Goor HV. Small bowel obstruction after total or subtotal colectomy: a 10-year retrospective review. Br J Surg. 1998;85(9):1242-5.

68. Parker MC, Ellis H, Moran BJ, Thompson JN, Wilson MS, Menzies D, et al. Postoperative adhesions: tenyear follow-up of 12,584 patients undergoing lower abdominal surgery. Dis Colon Rectum. 2001;44(6):822-9.

69. Parker MC, Wilson MS, Menzies D, Sunderland G, Clark DN, Knight AD, et al. The SCAR-3 study: 5year adhesion-related readmission risk following lower abdominal surgical procedures. Colorectal Dis. 2005;7(6):551-8.

70. Parulkar BG, Supe AN, Vora IM, Mathur SK. Effects of experimental non-closure of peritoneum on development of suture line adhesions and wound strength in dogs. Indian J Gastroenterol. 1986;5(4):251-3.

71. Pellicano M, Guida M, Bramante S, Acunzo G, DiSpiezio SA, Tommaselli GA, et al. Reproductive outcome after autocrosslinked hyaluronic acid gel application in infertile patients who underwent laparoscopic myomectomy. PLoS One. 2015;10(5):0125610

72. Pellicano M, Bramante S, Guida M, Bifulco G, DiSpiezio SA, Cirillo D, et al. Ovarian endometrioma: postoperative adhesions following bipolar coagulation and suture. Fertil Steril. 2008;89(4):7969.

73. Polymeneas G, Theodosopoulos T, Stamatiadis A, Kourias E. A comparative study of postoperative adhesion formation after laparoscopic vs open cholecystectomy. Surg Endosc. 2001;15(1):41-3.

74. Roy KK, Baruah J, Moda N, Kumar S. Evaluation of unilateral versus bilateral ovarian drilling in clomiphene citrate resistant cases of polycystic ovarian syndrome. Arch Gynecol Obstet. 2009;280(4):573-8.

75. Royal College of Obstetricians and Gynecologists, 2002 Peritoneal Closure. Clinical Green Top Guidelines, Guideline No. 15

76. Sawada T, Nishizawa H, Nishio E, Kadowaki M. Postoperative adhesion prevention with an oxidized regenerated cellulose adhesion barrier in infertile women 195. J Reprod Med. 2000;45(5):387-9.

77. Schreinemacher MH, Broek RP, Bakkum EA, Goor HV, Bouvy ND. Adhesion awareness: a national survey of surgeons. World J Surg. 2010;34(12):280512. 
78. Sharma M, Kriplani A, Agarwal N. Laparoscopic bipolar versus unipolar ovarian drilling in infertile women with resistant polycystic ovarian syndrome: a pilot study. J Gynecol Surg. 2006;22:105-11.

79. Stocchi L, Milsom JW, Fazio VW. Long-term outcomes of laparoscopic versus open ileocolic resection for Crohn's disease: follow-up of a prospective randomized trial. Surgery. 2008;144(4):622-7.

80. Takahashi K, Kita N, Kimura F, Fujiwara M, Noda Y, Miyazaki K. A comparative study using early secondlook laparoscopic evaluation of postoperative adhesion formation between two surgical procedures for polycystic ovarian syndrome. Obstet Gynecol Surv. 2007;62:443-5.

81. Talwar S, Laddha BL, Jain S, Prasad P. Choice of incision in surgical management of small bowel perforations in enteric fever. Trop Gastroenterol. 1997;18(2):78-9.

82. Taylor GW, Jayne DG, Brown SR, Thorpe H, Brown JM, Dewberry SC, et al. Adhesions and incisional hernias following laparoscopic versus open surgery for colorectal cancer in the CLASICC trial. Br J Surg. 2010;97:70-8.

83. Broek RP, Wilbers J, Goor HV. Electrocautery causes more ischemic peritoneal tissue damage than ultrasonic dissection. Surg Endosc. 2011;25(6):182734.

84. Tulandi T. Salpingo-ovariolysis: a comparison between laser surgery and electrosurgery. Fertil Steril. 1986;45(4):489-91.

85. Tulandi T, Al-Jaroudi D. Nonclosure of peritoneum: a reappraisal. Am J Obstet Gynecol. 2003;189(2):60912.

86. Tulandi T, Guralnick M. Treatment of tubal ectopic pregnancy by salpingotomy with or without tubal suturing and salpingectomy. Fertil Steril. 1991;55(1):53-5.

87. Krabben AAVD, Dijkstra FR, Nieuwenhuijzen M, Reijnen MM, Schaapveld M, Goor HV. Morbidity and mortality of inadvertent enterotomy during adhesiotomy. Br J Surg. 2000;87:467-71.

88. Goor HV. Consequences and complications of peritoneal adhesions. Colorectal Dis. 2007;9(2):2534.

89. Wong SS, Wilczynski NL, Haynes RB. Comparison of top-performing search strategies for detecting clinically sound treatment studies and systematic reviews in MEDLINE and EMBASE. J Med Libr Assoc. 2006;94(4):451-5.

Cite this article as: Rani R, Singh S, Urvashi, Garg R. Using different surgical techniques and ideas to reduce post-operative adhesion formation: a systematic review and meta-analysis. Int J Reprod Contracept Obstet Gynecol 2021;10:3560-74. 